\title{
THE FIRST MEASUREMENT OF THE ADIABATIC INDEX IN THE SOLAR CORONA USING TIME-DEPENDENT SPECTROSCOPY OF HINODE/EIS OBSERVATIONS
}

\author{
Tom Van Doorsselaere ${ }^{1,4}$, Nick Wardle $^{1}$, Giulio Del Zanna ${ }^{2}$, Kishan Jansari ${ }^{1}$, Erwin Verwichte ${ }^{1}$, \\ AND VALERY M. NAKARIAKOV ${ }^{1,3}$ \\ ${ }^{1}$ CFSA, Physics Department, University of Warwick, Coventry CV4 7AL, UK; Tom.VanDoorsselaere@wis.kuleuven.be \\ ${ }^{2}$ DAMTP, Centre for Mathematical Sciences, Wilberforce Road, Cambridge CB3 0WA, UK \\ ${ }^{3}$ Central Astronomical Observatory at Pulkovo of RAS, 196140 St. Petersburg, Russia \\ Received 2010 August 31; accepted 2010 November 22; published 2011 January 10
}

\begin{abstract}
We use observations of a slow magnetohydrodynamic wave in the corona to determine for the first time the value of the effective adiabatic index, using data from the Extreme-ultraviolet Imaging Spectrometer on board Hinode. We detect oscillations in the electron density, using the CHIANTI atomic database to perform spectroscopy. From the time-dependent wave signals from multiple spectral lines the relationship between relative density and temperature perturbations is determined, which allows in turn to measure the effective adiabatic index to be $\gamma_{\text {eff }}=1.10 \pm 0.02$. This confirms that the thermal conduction along the magnetic field is very efficient in the solar corona. The thermal conduction coefficient is measured from the phase lag between the temperature and density, and is shown to be compatible with Spitzer conductivity.
\end{abstract}

Key words: Sun: corona - Sun: oscillations - techniques: spectroscopic

\section{INTRODUCTION}

In analytical and numerical models of the solar and stellar coronae and other natural plasma systems, the adiabatic index $\gamma$ plays a crucial role in the hydrodynamic and thermodynamic description, e.g., relating the density and pressure. Often, an adiabatic relationship with $\gamma=5 / 3$ is postulated to govern the energetics of the considered mono-atomic plasma. Accounting for more complicated physics (e.g., radiative cooling or thermal conduction) requires the knowledge of the adiabatic index too.

In this Letter, we use data from the Extreme-ultraviolet Imaging Spectrometer (EIS; Culhane et al. 2007) on board the Japanese Hinode satellite (Kosugi et al. 2007), to observe propagating slow waves. EIS observations of waves (Van Doorsselaere et al. 2008b; Erdélyi \& Taroyan 2008; Wang et al. 2009b; Marsh $\&$ Walsh 2009; Mariska \& Muglach 2010) open up perspectives for combined seismological-spectroscopic plasma diagnostics. Here, we report the spectroscopic measurement of the temperature and density dynamics in a coronal loop. The comparison of these two measurements leads to the first measurement of the effective adiabatic index in the solar corona. The effective adiabatic index contains information about the thermal properties of the coronal plasma, and thus about the coronal heating function.

We obtain these results by the technique of coronal seismology (Roberts et al. 1983), which through the matching of observations with magnetohydrodynamic (MHD) theory of waves in structured plasmas allows for the measurement of local physical quantities. In the last decade, coronal seismology has been successful in determining the coronal magnetic field (Nakariakov et al. 1999), the coronal density stratification (Andries et al. 2005), and transverse structuring (Aschwanden et al. 2003; Van Doorsselaere et al. 2008a). Recently, it was conclusively shown that the solar corona and chromosphere are filled with ubiquitous transverse waves (Tomczyk et al. 2007; De Pontieu et al. 2007), opening up an enormous potential for the use of coronal seismology.

\footnotetext{
4 Now at Centrum voor Plasma-Astrofysica, Mathematics Department, KULeuven, Celestijnenlaan 200B bus 2400, 3001 Leuven, Belgium.
}

Slow magneto-acoustic modes can be observed as both periodic variations in intensity and Doppler shift provided that the loop axis has a large component along the line of sight. Such oscillations have been studied intensively. For example, perturbations in velocity and intensity have been observed using Solar and Heliospheric Observatory (SOHO) in hot coronal lines, Fe XIX and Fe XXI (Wang et al. 2002, 2003b). These were interpreted as standing magneto-acoustic waves due to the quarter period phase shift between velocity and intensity oscillations. Investigations with $\mathrm{SOHO} /$ Extreme-ultraviolet Imaging Telescopes $195 \AA$ data (Berghmans \& Clette 1999) and Transition Region and Coronal Explorer (TRACE) $171 \AA$ data (De Moortel et al. 2002) have reported the presence of propagating slow magneto-acoustic modes with periods of around 5 minutes. In studies by King et al. (2003) and Robbrecht et al. (2001), intensity disturbances were detected simultaneously in both $171 \AA$ and $195 \AA$ spectral lines using a combination of TRACE and $\mathrm{SOHO}$. The disturbances were interpreted as slow magnetoacoustic modes, due to their propagation speeds being lower than the local sound speed, propagating along magnetic field lines away from an active region.

\section{DATA ANALYSIS AND RESULTS}

The data analyzed here is taken on the 8th of 2007 February near the West limb of the solar disk starting at 13:06 UT. The EIS slit crosses a region of increased brightness, which is interpreted to be the footpoints of active region loops. This is displayed in Figure 1 where an overlay with a simultaneous TRACE image is shown.

The data have been processed using the standard SolarSoft routines eis_prep with default options. Using the Gaussian fitting routine eis_auto_fit, we obtain intensity and line-ofsight velocity time series. The velocities were further corrected for orbital motion through use of the routine eis_wave_corr.

EIS is known to suffer from instrumental jitter during sit-andstare campaigns. To examine the jitter effect on the EIS data, we have followed the procedure detailed in Wang et al. (2009a), where they construct a time series of the intensity along the slit. 


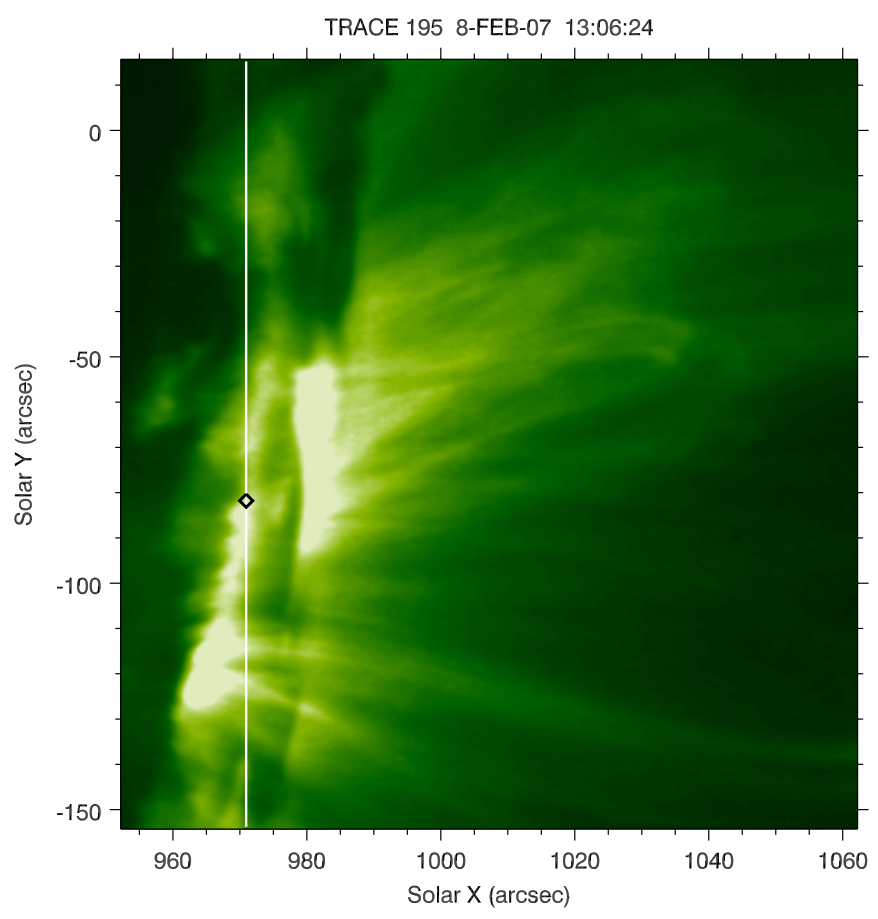

Figure 1. Solar coronal intensity map taken by TRACE. The solid white line indicates the position of the observational slit of EIS during the period studied while the black diamond indicates the macro pixel used.

Jitter would be visible as "dips" in this $Y-T$ diagram. Since no sudden "dips" were observed, we concluded that any vertical jitter is less than 3 ". Additionally, we investigated the jitter using the routine xrt_jitter and eis_jitter. This confirms the earlier result that the jitter is negligible during the current observation.

At the location of the diamond in Figure 1, an oscillatory signal is detected in both the intensity and Doppler velocity of the Fe XII $195 \AA$ spectral line. The time signal is displayed in Figure 2. We have eliminated the instrumental jitter as likely cause of the observed oscillation.

We find a period of $P_{V}=314 \pm 83 \mathrm{~s}$ and $P_{I}=344 \pm 61 \mathrm{~s}$ in the velocity and intensity, respectively. The fact that the oscillation is seen in both the velocity and intensity allows for a confident mode identification. The only coronal MHD oscillations that perturb significantly both the intensity and velocity without a noticeable change in the geometry are the sausage and the slow modes (Nakariakov \& Verwichte 2005). Here, the former mode can be disregarded because it cannot explain the 5 minute periodicity (Pascoe et al. 2007). Figure 2 shows that the oscillations in the intensity and velocity are in phase (blueshift corresponds to intensity decrease), which points to a running slow wave (a standing slow wave would have a quarter phase shift between the two quantities; Sakurai et al. 2002; Wang et al. 2003a). In this case, the maximumcorrelation time lag corresponds to $6 \mathrm{~s}$, which is much smaller than the period.

From a comparison of the velocity and intensity amplitude, it is possible to find the projected phase speed of the wave, which is smaller than $10 \mathrm{~km} \mathrm{~s}^{-1}$ in this case. Since this is much smaller than the accepted value for the sound speed (a couple of $100 \mathrm{~km} \mathrm{~s}^{-1}$ ), we conclude that the observed structure is nearly perpendicular to the line of sight. This is compatible with the observational conditions (Figure 1).

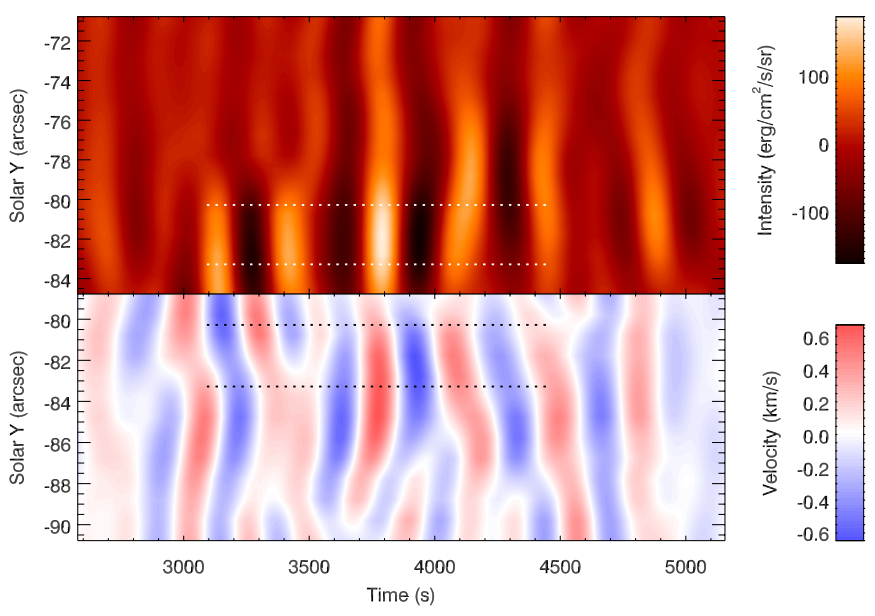

Figure 2. $Y-T$ diagram of the intensity (top) and the velocity (bottom) as observed in the Fe XII $195 \AA$ spectral line. The horizontal axis is time and the vertical axis is the distance along the EIS observing slit. Each time series has been filtered for oscillations with frequencies between $2.5 \mathrm{mHz}$ and $5 \mathrm{mHz}$ (top-hat filter between those limits). The extent of the studied pixel is indicated by horizontal dashed lines.

In the same observations, EIS is simultaneously taking data in different spectral windows. The wave is also visible in the Fe XIII $202 \AA$ and $203 \AA$ spectral lines. These lines, together with the Fe XII $195 \AA$ spectral line, are excellently suited for spectroscopic analysis. We use the CHIANTI (Dere et al. 1997) software to determine the density from the line ratio of the two Fe XIII spectral lines (assuming a constant temperature) and the temperature from the line ratio of the Fe XIII $202 \AA$ and Fe XII $195 \AA$ spectral lines (while taking into account the density variations). The time series for the density and temperature are shown in Figures 3(A) and (B). It is evident that the electron density is oscillating in phase with the intensity (as expected). The temperature variations follow roughly the same trend.

From linearized ideal MHD theory (e.g., Goossens 2003), we know that

$$
\frac{\rho^{\prime}}{\rho_{0}}=\frac{1}{\gamma_{\mathrm{eff}}} \frac{p^{\prime}}{p_{0}}=\frac{1}{\gamma_{\mathrm{eff}}-1} \frac{T^{\prime}}{T_{0}},
$$

where $\rho, p, T$ are, respectively, the mass density, the gas pressure, and the temperature. A superscript ' indicates perturbed quantities and a subscript ${ }_{0}$ stands for equilibrium quantities. To obtain this equation, we have assumed that the energy equation may be expressed through a polytropic relation $p=K \rho^{\gamma_{\text {eff }}}$, with an effective adiabatic index $\gamma_{\text {eff }}$. This equation describes a linear relationship between the observables $\left(\rho^{\prime} / \rho_{0}, T^{\prime} / T_{0}\right)$. We have used least-squares to fit a line to the scatter plot (Figure 3(C)) of the observational data of these quantities. We find that $\gamma_{\text {eff }}=1.10 \pm 0.02$. This is the first measurement of the adiabatic index in the corona. The uncertainty on $\gamma_{\mathrm{eff}}$ has been calculated through propagating the photon noise of the observations. Due to uncertainties in the spectroscopic data and background subtraction, it is impossible to rule out the possibility of an isothermal corona, though.

\section{IMPLICATIONS}

This first measurement of the effective adiabatic index has important implications for solar coronal physics and modeling. First and foremost, the fact that the effective adiabatic index is different from $5 / 3$ (the adiabatic index in a mono-atomic gas) means that the energy equation in the MHD equations cannot be represented with an adiabatic form supplemented 

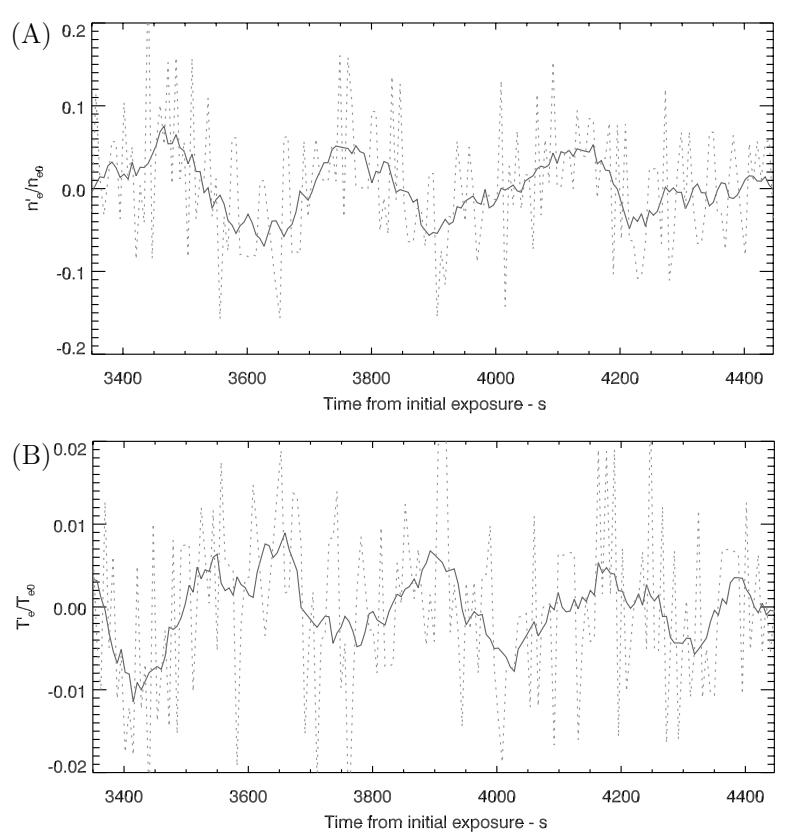

(C)
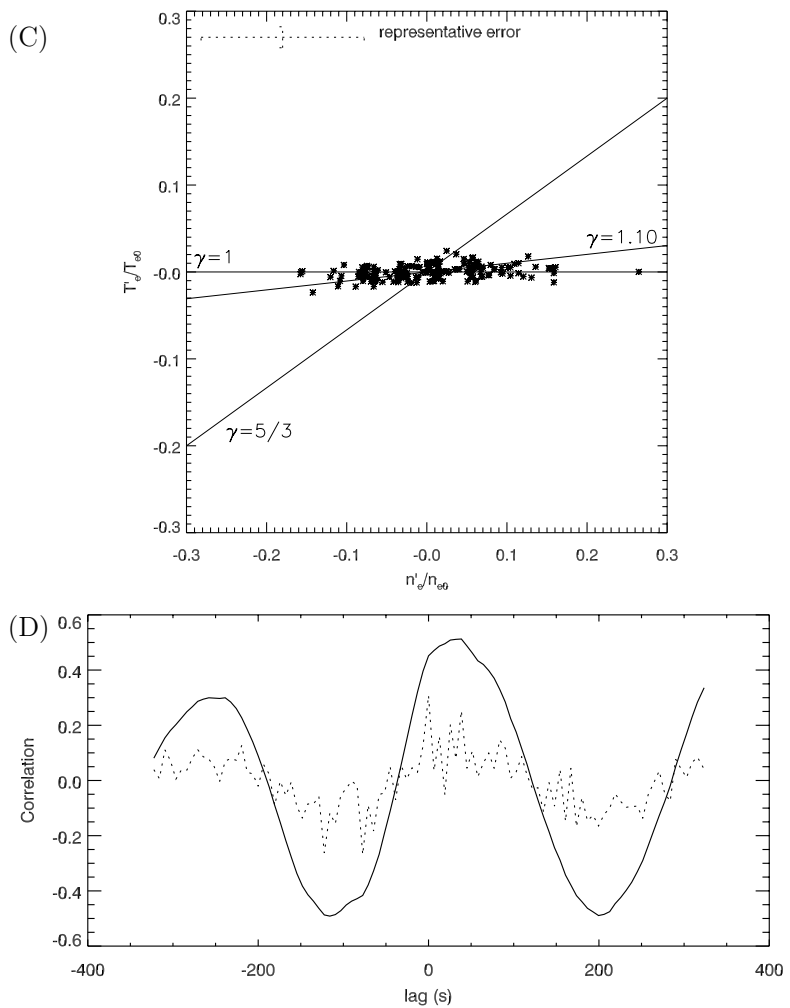

Figure 3. $n_{\mathrm{e}}^{\prime} / n_{\mathrm{e} 0}(\mathrm{~A})$ and $T_{\mathrm{e}}^{\prime} / T_{\mathrm{e} 0}$ (B) vs. time, as derived from the line ratios of Fe XIII $202 \AA$ and $203 \AA$, and Fe XII $195 \AA$ and Fe XIII $202 \AA$. The full line shows the smoothed time series, whereas the dotted lines show the raw spectroscopic data. Panel (C) shows the scatter plot of the electron density and temperature (crosses), with the best-fitting line and the classical values of $\gamma=1$ and $\gamma=5 / 3$ overlaid. Panel (D) displays the correlation between the density and temperature as a function of lag. The solid line is the correlation between the smoothed time series, and the dashed line is between the raw time series.

with small correction terms for energy gains and losses. Thermal conduction, radiative losses, or turbulence must be important to lower the effective adiabatic index to the observed value. Indeed, the low amplitude of the temperature variations suggest that the thermal conduction is very efficient. This agrees with earlier theoretical work (De Moortel \& Hood 2003).
It is possible to go a step further, using the assumption that the observed wave is a propagating slow wave. It has been shown previously (Owen et al. 2009) that the thermal conduction introduces a phase shift between the density and temperature perturbations. This phase shift $\phi$ can be theoretically calculated to be

$$
\tan \phi=\frac{k^{2}(\gamma-1) \kappa_{\|} T_{0}}{p_{0} \omega},
$$

where $\kappa_{\|}=\kappa_{0} T_{0}^{5 / 2}$ is the parallel thermal conduction. It has been assumed that the density and temperature perturbations follow $\exp i(\omega t-k z)$ with frequency $\omega$ and wavenumber $k . t$ stands for time and $z$ is the direction along the magnetic field. The spectroscopic measurements of the density and temperature are $n_{\mathrm{e} 0}=1.710^{15} \mathrm{~m}^{-3}$ and $T_{0}=1.7 \mathrm{MK}$, respectively.

To measure the observed phase shift, we have plotted the correlation between the temperature and density as a function of the phase lag between them (Figure 3(D)). The correlation peaks for a lag of $40 \mathrm{~s}$, corresponding to $\phi \approx 50^{\circ}$. Using the measured values of the temperature, density, and period, the phase lag leads to a value of $\kappa_{0}=9 \times 10^{-11} \mathrm{~W} \mathrm{~m}^{-1} \mathrm{~K}^{-1}$ (taking $\gamma=1.1$ ) or $\kappa_{0}=2 \times 10^{-11} \mathrm{~W} \mathrm{~m}^{-1} \mathrm{~K}^{-1}$ (taking $\gamma=5 / 3)$. These values for the thermal conduction are of the same order of magnitude as the classical values for $\kappa_{0}$ of $10^{-11} \mathrm{~W} \mathrm{~m}^{-1} \mathrm{~K}^{-1}$ (Priest 1984) for Spitzer conductivity. This experimentally justifies the applicability of this value in the modeling of field-aligned thermal conduction in the corona.

Of course, in the case that the thermal conduction is strong, Equation (1) is not valid anymore. It can be shown from Equation (11) in De Moortel \& Hood (2003) that the adiabatic index can be calculated from

$$
A_{\mathrm{T}}=\cos \phi(\gamma-1) A_{\rho},
$$

when the thermal conduction is not negligible. $A_{\mathrm{T}}$ and $A_{\rho}$ stand for the relative oscillation amplitudes of the temperature and the density. Such a self-consistent determination of the adiabatic index leads to a value of $\gamma=1.17$ in this case. The fact that the adiabatic index is still significantly different from 5/3 after taking into account the thermal conduction means that other terms in the energy equation play an important role, such as the radiative cooling or coronal heating term.

\section{CONCLUSIONS}

Hinode/EIS observations enable the possibility of doing spectroscopic time-dependent coronal seismology. This new technique allowed for the first determination of the effective adiabatic index in the solar corona, which is calculated to be $\gamma_{\text {eff }}=1.10 \pm 0.02$. This value contains information about the thermal properties of the solar coronal plasma. The fact that it is close to unity suggests that the thermal conduction is important, as has been found in previous studies as well. We have also calculated the thermal conduction coefficient from the phase difference between the temperature and density, obtaining a value of the same order of magnitude as the coronal thermal conduction coefficient in classical Spitzer conductivity.

The fact that the adiabatic index is not close to the commonly used value of 5/3 means that other terms in the energy equation are important. Moreover, taking an adiabatic relationship with the measured adiabatic index in a numerical code for the modeling of the solar corona allows for an ad hoc description of the plasma energy equation, which includes the unknown coronal heating function. 
The authors thank Professor A. Hood, Dr. G. Doschek, and Dr. C. Foullon for constructive comments to early drafts of this manuscript. T.V.D. has received funding from the European Community's seventh framework programme (FP7/2007-2013) under grant agreement number 220555. T.V.D is a postdoctoral fellow of the FWO-Vlaanderen. E.V. acknowledges the financial support from the Engineering and Physical Sciences Research Council (EPSRC) Science and Innovation award.

Facilities: Hinode, TRACE

\section{REFERENCES}

Andries, J., Arregui, I., \& Goossens, M. 2005, ApJ, 624, L57

Aschwanden, M. J., Nightingale, R. W., Andries, J., Goossens, M., \& Van Doorsselaere, T. 2003, ApJ, 598, 1375

Berghmans, D., \& Clette, F. 1999, Sol. Phys., 186, 207

Culhane, J. L., et al. 2007, Sol. Phys., 243, 19

De Moortel, I., \& Hood, A. W. 2003, A\&A, 408, 755

De Moortel, I., Hood, A. W., Ireland, J., \& Walsh, R. W. 2002, Sol. Phys., 209, 89

De Pontieu, B., et al. 2007, Science, 318, 1574

Dere, K. P., Landi, E., Mason, H. E., Monsignori Fossi, B. C., \& Young, P. R. 1997, A\&AS, 125, 149

Erdélyi, R., \& Taroyan, Y. 2008, A\&A, 489, L49

Goossens, M. 2003, An Introduction to Plasma Astrophysics and Magnetohydrodynamics (Dordrecht: Kluwer)

King, D. B., Nakariakov, V. M., Deluca, E. E., Golub, L., \& McClements, K. G. 2003, A\&A, 404, L1
Kosugi, T., et al. 2007, Sol. Phys., 243, 3

Mariska, J. T., \& Muglach, K. 2010, ApJ, 713, 573

Marsh, M. S., \& Walsh, R. W. 2009, ApJ, 706, L76

Nakariakov, V. M., Ofman, L., DeLuca, E. E., Roberts, B., \& Davila, J. M. 1999, Science, 285, 862

Nakariakov, V. M., \& Verwichte, E. 2005, Living Rev. Sol. Phys., 2, 3

Owen, N. R., De Moortel, I., \& Hood, A. W. 2009, A\&A, 494, 339

Pascoe, D. J., Nakariakov, V. M., \& Arber, T. D. 2007, A\&A, 461, 1149

Priest, E. R. 1984, Solar Magneto-hydrodynamics (Geophysics and Astrophysics Monographs; Dordrecht: Reidel)

Robbrecht, E., Verwichte, E., Berghmans, D., Hochedez, J. F., Poedts, S., \& Nakariakov, V. M. 2001, A\&A, 370, 591

Roberts, B., Edwin, P. M., \& Benz, A. O. 1983, Nature, 305, 688

Sakurai, T., Ichimoto, K., Raju, K. P., \& Singh, J. 2002, Sol. Phys., 209, 265

Tomczyk, S., McIntosh, S. W., Keil, S. L., Judge, P. G., Schad, T., Seeley, D. H., \& Edmondson, J. 2007, Science, 317, 1192

Van Doorsselaere, T., Brady, C. S., Verwichte, E., \& Nakariakov, V. M. 2008a, A\&A, 491, L9

Van Doorsselaere, T., Nakariakov, V. M., Young, P. R., \& Verwichte, E. 2008b, A\&A, 487, L17

Wang, T., Solanki, S. K., Curdt, W., Innes, D. E., \& Dammasch, I. E. 2002, ApJ, 574, L101

Wang, T. J., Ofman, L., \& Davila, J. M. 2009a, ApJ, 696, 1448

Wang, T. J., Ofman, L., Davila, J. M., \& Mariska, J. T. 2009b, A\&A, 503, L25

Wang, T. J., Solanki, S. K., Innes, D. E., Curdt, W., \& Marsch, E. 2003a, A\&A, 402, L17

Wang, T. J., Solanki, S. K., Curdt, W., Innes, D. E., Dammasch, I. E., \& Kliem, B. 2003b, A\&A, 406, 1105 